\title{
A User-Centered Interface for Querying Distributed Multimedia Databases
}

\author{
Isabel F. Cruz \\ Kimberly M. James \\ Worcester Polytechnic Institute \\ Department of Computer Science \\ 100 Institute Road, Worcester, MA 01609 \\ \{ifc, beez\}@cs.wpi.edu
}

\begin{abstract}
Facilitating information retrieval in the vastly growing realm of digital media has become increasingly difficult. Delaunay ${ }^{\mathrm{MM}}$ seeks to assist all users in finding relevant information through an interactive interface that supports pre- and post-query refinement, and a customizable multimedia information display. This project leverages the strengths of visual query languages with a resourceful framework to provide users with a single intuitive interface. The interface and its supporting framework are described in this paper.
\end{abstract}

\section{Keywords}

Multimedia Querying, Customizable User Interfaces, Distributed Database Access

\section{INTRODUCTION}

With increasingly diverse user populations and available technologies on the World Wide Web, the value of reliable searching and information navigation mechanisms is becoming more widely recognized. To facilitate accurate retrieval of information, search interfaces must combine powerful search and refinement capabilities with simple views so as not to overwhelm users.

Delaunay $^{\mathrm{MM}}$ combines a simple interface with the advanced refinement and customization features offered through visual querying, to provide users with a unique interface for query specification and refinement, and for displaying the multimedia objects that are the answers to the queries $[1,2]$. These objects are laid out in virtual documents that are user-specified. Our current work focuses on increasing the usability of the user interface to Delaunay $^{\mathrm{MM}}$. To this end, we have introduced significant changes to both the previous interface and the underlying framework $[1,2]$, which we describe in this paper. The current prototype was developed in Java.

Permission to make digital or hard copies of all or part of this work for personal or classroom use is granted without fee provided that copies are not made or distributed for profit or conmercial advantage and that copies bear this notice and the full citation on the first page. To copy otherwise, to republish, to post on servers or to redistribute to lists. requires prior specific permission and/or a fee.

SIGMOD '99 Philadelphia PA

Copyright A.CM 1999 1-58113-084-8/99/05 $\ldots \$ 5.00$

\section{USER INTERFACE}

The Delaunay ${ }^{\mathrm{MM}}$ user interface is comprised of four components that work in tandem to provide users with the requisite features. This interface is supported by an architecture, as discussed in Section 3, that passes information on to the interface modules. The primary modules support query specification and the display of the results, shown in Figures 2 and 3, with query refinement implemented by the intermediate modules. Each module transparently operates with its respective architectural component to provide powerful functionality via a simple interface.

\subsection{Query Specification}

On the initial screen (see Figure 2), the query keywords are specified and optional fields for customization are available. Keywords are entered as text, as in most engines, but unlike in most, the Boolean operators are provided. The operators are laid out to prevent their incorrect use and to eliminate the need for users to understand Boolean query construction.

The optional fields allow users to select the maximum number of objects to return, desired information sources, predefined page format (Section 2.3), type of objects to display, and level of interaction. Objects are of type text, image, audio, or video. Users that have saved searches may also select to return to their previous search results.

The defaults provided offer the most powerful customized interface for finding the smallest number of relevant results, from all available sources and object types, using a comprehensive results display interface. If users select the advanced features, they are able to create their own interface using the visual querying methods previously developed $[1,2]$, and refine their queries based on the attributes of the target information sources.

\subsection{Query Context and Refinement}

To increase the number of relevant results and decrease the query execution time, a pre-query refinement option, the Search Assistant, is provided. This assistant shows users the context in which their keywords are found using an ontological representation. This representation allows users to understand the context of their queries, while also relaying structural information about the available resources. Post-query refinement is provided when the number of returned results exceeds the number selected at query submission time. The refinement interface displays all

\footnotetext{
* Research supported in part by the National Science Foundation under CAREER Award IRI-9896052 and CISE Research Instrumentation Grant 9729878.
} 
returned objects in the left column, and associated information in the right. This information includes the object in full, for example, displaying full text for text objects, and object metadata, such as ownership information and associated keywords. Through this interface, users may select representative objects to use for refinement, similar to a collective "More like this option", or to use in creating the virtual page.

This interface's strength is in its ability to display all search results and in allowing users to select the objects that are of interest to them, thus effectively facilitating user-centered refinement.

\subsection{Virtual Document Display}

The virtual document display is used to present users' query results in a single format that users can browse without leaving the Delaunay ${ }^{\mathrm{MM}}$ site. Delaunay ${ }^{\mathrm{MM}}$ also supports a "Go to Web Page" option, that allows users to go to the original web page. This presentation method preserves the context of retrieved information, while optionally allowing users to view the original web page [3]

Pre-defined formats are designed based on task and user types. For example, the student/researcher format seeks to preserve the relationship between objects, as found on their owning pages, in order to provide complete sets of information. On the other hand, the comparison formats seek only to provide users with the returned objects positioned side by side, so that they may be compared to one another as they are traversed.

Before exiting Delaunay ${ }^{\mathrm{MM}}$ or a particular query, users may also select ("bookmark") the query results. This option allows for the results to be viewed at a later time; it is particularly useful when the refinement features are used to derive the query results, because subsequent queries are created by Delaunay ${ }^{\mathrm{M}}$ based on the selected refinement objects, which are not visible to the users.

\section{Delaunay ${ }^{\mathrm{MM}}$ ARCHITECTURE}

The necessary user interface information is provided by welldefined middle layer components. These components are assumed to have access to the following information:

1. Ontology/structure of information repositories.

2. Object metadata.

3. Spatial and contextual relationship between objects.

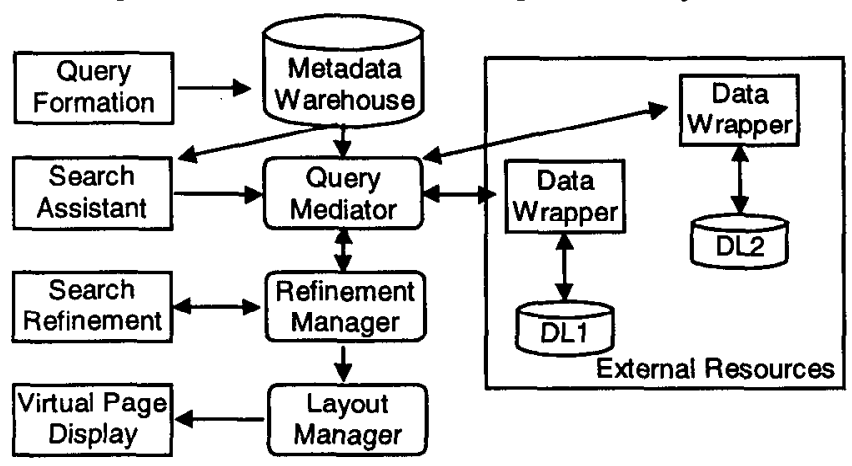

Figure 1: Delaunay ${ }^{\mathrm{MM}}$ Architecture

\subsection{Metadata Warehouse}

The metadata warehouse is a resident resource that provides Delaunay $^{\mathrm{MM}}$ with the metadata and structure associated with the information repositories. After the keywords are submitted, the metadata warehouse is consulted to determine in what context the keywords are found in the information repositories. A virtual ontology that represents globally the context of the keywords is subsequently built. This virtual ontology is displayed to the user via the Search Assistant for further selection. It is important to note that this resource only provides information about the contents of the information repositories and not their actual contents.

\subsection{Query Mediator}

The query mediator is the crux of the interaction, with the external resources supporting both the pre- and post-query refinement options. It sends and receives query information to and from the data wrappers for individual repository access.

The pre-query option uses the virtual ontology provided by the metadata warehouse, which includes information repository specifics, to determine which external resources to query. The query mediator then takes the results returned from these resources and passes them to the refinement manager.

When the refinement manager is utilized, the query mediator is again used to facilitate post-query refinement. Using the queries passed to it from the refinement manager, it accesses the appropriate information resources, and returns the results to the refinement manager. It further determines when resources may be excluded from searches based on the representative refinement objects.

\subsection{Refinement Manager}

After receiving the query results from the query mediator this component determines whether further refinement is needed by means of the user's selected number of objects to return. If the number returned is greater than the selected one, it passes the objects to the search refinement interface allowing users to select which objects to use for refinement or virtual document creation.

If refinement is selected, using the metadata associated with each object and a refinement algorithm, the manager creates, on the fly, a new query to send back to the query mediator. This new query combines the attributes of all representative objects.

\subsection{Layout Manager}

The layout manager creates the virtual document, as selected by the user, from the returned query results (see Figure 3). Currently, fixed-coordinate templates are used to determine the layout, but constraint-based mechanisms will be incorporated to generate the layout dynamically.

\section{USABILITY STUDY RESULTS}

In December 1998, 34 students participated in the first usability study of the new interface and architecture. This study focused on three components: the intuitiveness of the new interface, the applicability of the approach to querying vast data resources, and the identification of flaws in the approach and interface. The overall results of the study supported the approach and interface, suggesting minor changes that would significantly increase overall usability.

For this study, users were asked to use Delaunay ${ }^{\mathrm{MM}}$ to submit two queries to various digital resources, and to create their own virtual page format using the original application $[1,2]$. Each query used different Delaunay ${ }^{M M}$ attributes to tailor the interface to the specific task. Once finished, users were asked to complete a 40 - 
question survey in which they either affirmed or negated statements about each aspect of the interface. Two users were monitored to confirm that the study's usage scenarios and survey questions were fair. All other users performed the study over the course of a week for 30 to 45 minutes at their leisure. A detailed discussion of the results is available elsewhere [4].

The overall results of the study were very positive. Of the respondents, $59 \%$ were expert web searchers having high confidence in their searching capabilities, and all others were average. All respondents noted that they are typically successful in finding information on the Internet, with most (88\%) finding the necessary information within submitting a few searches. In response to the statement: "I would prefer this interface to existing search interfaces.", there was a close split between positive and negative responses (36\% and $40 \%$, respectively). Many of the users commented that the response time of this approach was too slow. This slow response time is a result of the need to transfer multimedia elements, as opposed to hypertext, over the Internet. Ultimately, for expert web searchers response time is a key usability factor [8], thus the interface was not regarded as highly by these individuals. We are currently investigating the optimization of the transfer of multimedia objects in response to different kinds of queries in Delaunay ${ }^{M M}$.

\section{RELATED WORK}

There are several projects underway that directly relate to our work in the following areas: interface design [11,13], query definition and submission $[9,10,12]$, ontological development $[5,6,15]$, database mediation [7,14], and data warehousing [16].

\section{CONCLUSIONS AND FUTURE WORK}

Delaunay $^{\mathrm{MM}}$ offers a unique user-centered approach to querying multimedia information repositories. The approach has been validated by a usability study, with another planned in the immediate future for a wider variety of users, including inexperienced ones. The framework and interface of Delaunay ${ }^{\text {MM }}$ offer a basis for fusing together existing efforts in digital library and in distributed multimedia databases research. This framework can be extended to support World Wide Web applications.

There are several areas for future exploration: ontological incorporation, intelligent mediation, multimedia retrieval optimization, and query tracking and reuse.

\section{REFERENCES}

[1] I.F. Cruz and W.T. Lucas. A Visual Approach to Multimedia Querying and Presentation. In Fifth ACM International Multimedia Conference, 1997, pp. 109-120.

[2] I. F. Cruz and W. T. Lucas. A Customizable Layout-Driven Approach to Querying Digital Libraries. SPIE Multimedia Computing and Networking, 1999, pp. 122-134.

[3] I.F. Cruz and K.M. James. Preserving Contextual Navigation in Hypermedia Querying. [Online] Available http://www.cs.wpi.edu/ beez/MEMEX.pdf, January 19, 1999.
[4] I.F. Cruz and K.M. James. A User Interface for Distributed Multimedia Database Querying with Mediator Supported Refinement. [Online] Available http://www.cs.wpi.edu/ - beez/IDEAS99.ps, January 22, 1999.

[5] R. Daniel Jr., C. Lagoze, and S.D. Payelle. A Metadata Architecture for Digital Libraries. Advances in Digital Libraries Conference, 1998. [On-line] Available: http:// dlib.computer.org/conferen/adl/8464/pdf/84640276.pdf.

[6] A. Farquhar, R. Fikes, W. Pratt, and J. Rice. Collaborative Ontology Construction for Information Integration. Knowledge Systems Laboratory, Stanford University, KSL95-63, August 1995.

[7] H. Garcia-Molina, Y. Papakonstantinou, D. Quass, A. Rajaraman, Y. Sagiv, J.D. Ullman, V. Vassalos, and J. Widom. The TSIMMIS Approach to Mediation: Data Models and Languages. In Journal of Intelligent Information Systems, 8(2): 117-132, 1997.

[8] Georgia Tech Research Corporation. GVU's 9th WWW User Survey. [Online] Available http://www.cc.gatech.edu/ gvu/user_surveys/survey-1998-04/, October 9, 1998.

[9] S. Greene, E. Tanin, C. Plaisant, and B. Shneiderman. The End of Zero-Hit Queries: Query Previews for NASA's Global Change Master Dictionary. [Online] Available ftp://ftp.cs.umd.edu/pub/hcil/Reports-AbstractsBibliography/4856HTML/4856.html, January 8, 1998.

[10] S. Jones. Dynamic Query Result Previews for a Digital Library. In Third ACM Conference on Digital Libraries, 1998, pp. 291 - 292.

[11] G. Marchionini, C. Plaisant, and A. Komlodi. Interfaces and Tools for the Library of Congress National Digital Library Program. [Online] Available ftp://ftp.cs.umd.edu/ pub/hcil/ReportsAbstractsBibliography/3872HTML/3872.ht ml, February 11, 1998.

[12] B. Shneiderman, D. Byrd, and W.B. Croft. Clarifying Search: A User-Interface Framework for Text Searches. [On-line] Available http://www.dlib.org/dlib/january97/ retrieval/01 shneiderman.html, January 15, 1997.

[13] J. Takahashi, T. Kushida, J. Hong, R. Rieger, W. Martin, G. Gay, S. Sugita, Y. Kurita, J. Reeve, R. Loverance. Global Digital Museum: Multimedia Information Access and Creation on the Internet. In Third ACM Conference on Digital libraries, 1998, pp. 244 - 253.

[14] A. Tomasic, L. Raschid, and P. Valduriez. Scaling Access to Heterogeneous Data Sources with DISCO. IEEE Transactions on Knowledge and Data Engineering, 10(5): 808-823, 1998.

[15] P. Weinstein. Ontology-Based Metadata: Transforming the MARC Legacy. In Third ACM Conference on Digital Libraries, 1998, pp. 254 - 263.

[16] J. Widom. Research Problems in Data Warehousing. In Fourth Conference on Information and Knowledge Management, Nov. 1995, pp. 25-30. 


\section{Welcome to DelaunayMM}

Search for information on:

OR

BUT NOT

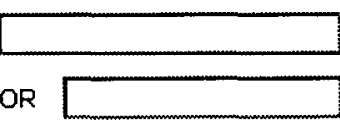

You may enter as many words as you like

Optionel Words used to BROADEN the search

Optional Words used to NARROW the search

Your Saved Searches:

You may select one of your
previous searches and press Search to seo results
- Optional Fields

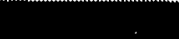

Maximum Number of Objects to Return: 25

Information Sources: All arailatale 9ustices

Perseus Project

Venetian Archives

Diatial Dinnsaurs

Select a Page Format StudentiResearcher

Display. $\nabla$ Images $\nabla$ Text $\nabla$ Audio $\bar{V}$ video

Use Advanced Search and Layout Features

Figure 2: Delaunay ${ }^{\mathrm{MM}}$ Query Specification

\section{DelaunayMM Search Results}

Student/Researcher Page Format

\section{1 (0)}
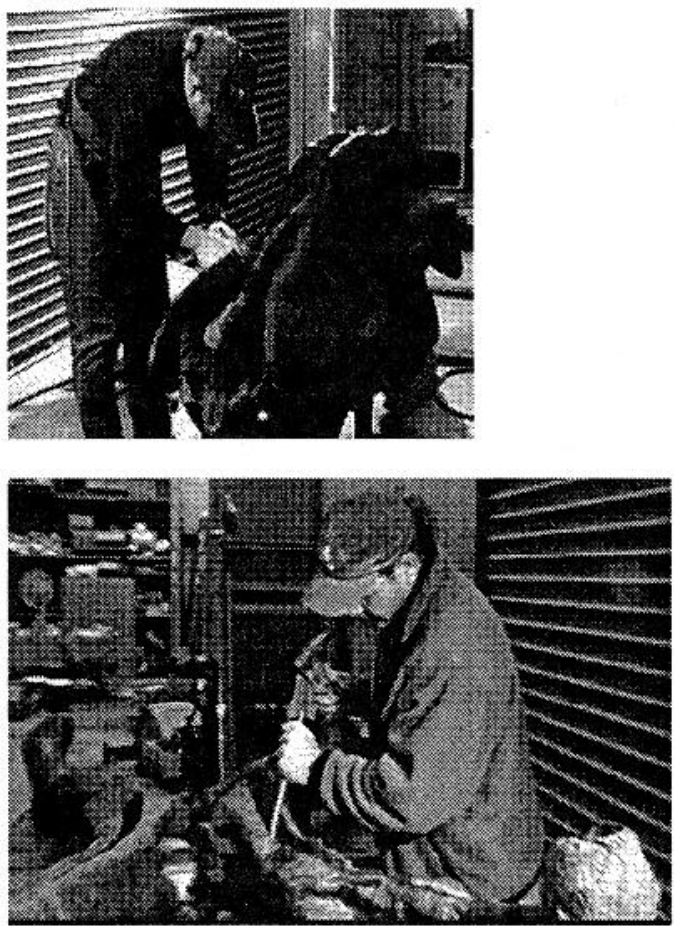

Nyoming Dinosaur Center paleontological technician Ed Cole prepares bones for mounting. $10 / 11 / 98$ WHO IS STAN? 'Stan' is the most complete fossil Tyrannosaurus rex ever found. T. rex lived in the Cretaceous period 83 to 65 million years ago in the area that is now Montana, wyoming, the Dakotas and parts of Canada. It is a theropod -- a meat-eater (carnivore) that walked on its back legs and had three of fewer working toes on each foot. In 1987, Stanley Sacrison, an amateur paleontologist, was working near Buffalo, South Dakota, when he spotted a large pelyis weathering out of a sandy cliff face 100 feet above the prairie. But it was not untll the spring of 1992 that excayation began by Black Hills In stitute of Geological Research, Hill City, South Dakota.The excavation was a major undertaking. Each area of the dig was carefully mapped before the fossil bones were removed. This allowed scientists to keep thorough recards of the find. Information such as where each bone is found and its condition can help scientists understand how and why the dinosaur died. The skeleton of Stan took more than 30,000 hours to prepare. $A$ copy (cast) of each bone was made. The original bones are at the Black Hills Museum of Natural History. Stan turned out to be the most complete T. rex skeleton ever found. Many dinosaur skeletons are found with no skull. But Stan had almost his entire skull - over 40 bones in all. The skull is five feet long and its teeth are almost five inches long. The life-size fossil skeleton at The wyoming Dinosaur Center is expected to be 40 feet long and between 11 and 15 feet hlgh at the shoulder. Stan must have led a very ferocious life. He had many face injurles, his neck and several ribs had been broken, two vertebrae had fused

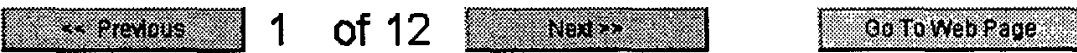

To retrieye these results, you may save them with a name Name that will appear the next time you visit Delaunayma
Not Pleased With These Results? Try Refining the Search

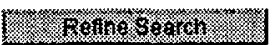

Figure 3: Delaunay ${ }^{\mathrm{MM}}$ Student/Researcher Virtual Document

(Text and pictures printed with the permission of the Wyoming Dinosaur Center, http://wyodino.org/) 